A. Weisbecker, M. Burmester \& A. Schmidt (Hrsg.): Mensch und Computer 2015 Workshopband, Stuttgart: Oldenbourg Wissenschaftsverlag, 2015, S. 109-115.

\title{
VODINO - Validierung und Optimierung des individuellen Nutzens von Ortungssystemen bei Demenz
}

\author{
Herlind Megges ${ }^{1}$, Torsten Grewe ${ }^{1,2}$, Oliver Peters ${ }^{1}$ \\ Charité Universitätsmedizin Berlin, Klinik für Psychiatrie, Modul Altersmedizin ${ }^{1}$ \\ Hochschule für Technik und Wirtschaft, Berlin²
}

\section{Zusammenfassung}

In diesem Beitrag wird ein Forschungsprojekt vorgestellt, welches die Validierung und Optimierung des individuellen Nutzens von Ortungssystemen (VODINO) für Menschen mit Demenz und deren pflegende Angehörige zum Ziel hat. Bisher werden technische Lösungen im Bereich Ortung bei Demenz noch wenig genutzt. Das Projekt soll wesentliche Schritte zur Verbesserung bereits bestehender Ortungssysteme liefern sowie Handlungshinweise und Werkzeuge für ein optimiertes Vorgehen bei der Auswahl und Implementierung dieser technischen Hilfen geben. Die Nutzerintegrierung in verschiedenen Schritten des Projektes steht im Vordergrund. In einem ersten Schritt wird die Frage bearbeitet welche potentiellen Nutzer der Zielgruppe von welchem Ortungssystem profitieren können. Das Ergebnis soll eine in der Praxis anwendbare Checkliste sein. Es wird diskutiert wie ein Mehrwert für Menschen mit Demenz und deren Angehörige im Zusammenspiel zwischen den spezifischen Bedürfnissen der Zielgruppe, dem Nutzungserlebnis und den Produkten selbst erreicht werden kann.

\section{Einleitung}

Die Zahl der Menschen mit Demenz in Deutschland wird weiter steigen. Schon jetzt sind es ca. eineinhalb Millionen Erkrankte, von denen der Großteil in der eigenen Häuslichkeit lebt. Aus Daten des Berlin-Institutes für Bevölkerung und Entwicklung (2012) sowie des Bundesministeriums (Schneekloth \& Wahl, 2005) geht hervor, dass es in Deutschland derzeit ca. eine Millionen Pflegende von Menschen mit Demenz im häuslichen Umfeld gibt. Dort werden sie vorwiegend von ihren Ehepartnern betreut (ebd.). In der aktuellen politischen 
Diskussion wird vielfach die Frage diskutiert, wie in Zukunft die Betreuung und Pflege von Menschen mit Demenz optimiert werden kann. Deshalb besteht große Notwendigkeit Pflegenden und Betroffenen zu vermitteln, dass sie in ihrer Situation nicht alleine sind und mit all ihren Sorgen und Bedürfnissen wahrgenommen werden. Die aktuellen Neuerungen innerhalb der Pflegeversicherung fokussieren diese Gruppe allerdings nur am Rande und weniger durch konkrete Unterstützungsangebote. Um das objektive und subjektive Sicherheitsbedürfnis der Zielgruppe zu adressieren ist die Möglichkeit der Ortung einer der wichtigsten Bereiche im Kontext von Demenz und Technik, da insbesondere Orientierungsschwierigkeiten in mehreren und gerade auch in frühen Stadien der Demenzerkrankung eines der Hauptsymptome darstellen.

\section{Theoretischer Hintergrund}

Im Folgenden werden der Stand der Forschung zur Thematik sowie eigene Vorarbeiten skizziert.

\subsection{Stand der Forschung}

Der hohe Bedarf seitens der Betroffenen ist seit einigen Jahren bekannt (Kinney et al., 2003; Braun et al., 2010). Der technische Fortschritt hat es möglich gemacht, Lösungen für die Privatverbraucher anzubieten. Die auf dem Markt befindlichen Produkte wurden in der Vergangenheit bereits kritisch evaluiert (z.B. Wan et al., 2014; Sapmaz et al., 2012; Sapmaz, 2012). Die drei Hauptgründe, welche für Ortungslösungen in dem Bereich sprechen, werden von Vuong und Kollegen (2015) wie folgt zusammengefasst: „Managing wandering behavior of people with dementia (PWD) has become increasingly imperative for these reasons: its high prevalence (60\%) among PWD, its negative outcomes such as falls or elopement, and its burden on caregivers." Für diese Zielgruppe sichere Mobilität zu ermöglichen und Monitoring Technologien $\mathrm{zu}$ entwickeln, stellt insgesamt ein rasch wachsendes Forschungsfeld dar. $\mathrm{Zu}$ diesem Schluss gelangen die Autoren Altendorf und Schreiber (2015) anhand einer durchgeführten Metaanalyse zahlreicher Studien aus diesem spezifischen Feld. Sie betonen auch: „statistical evidence for the effectiveness of safer walking and monitoring devices in dementia care is still lacking. “

\subsection{Eigene Vorarbeiten}

Unsere Forschungsgruppe befasst sich bereits seit mehreren Jahren mit der Thematik. Das von uns als wissenschaftliche Einrichtung begleitete ProVIEL-Projekt der Investitionsbank des Landes Brandenburg (Förderkennzeichen: 80153908) entwickelte 2013 in einem ersten Schritt ein Ortungssystem mit dem Namen Digital Care Support - DCS nahe am Anwender und dessen Bedürfnissen (Megges et al., 2013(b)). Der Fokus lag dabei auf der Softwareentwicklung. Als Hardware wurden herkömmliche Smartphones verwendet. In der sich anschließenden vierwöchigen Nutzerstudie mit achtzehn Dyaden von Menschen mit Demenz und deren pflegenden Angehörigen konnte vor allem gezeigt werden, dass die 
Hardware für den Menschen mit Demenz insuffizient ist (Megges 2014 (b)). Die Software hingegen wurde im Durchschnitt trotz kleiner Problembereiche als gut eingestuft. Mittlerweile bietet der freie Markt diverse Ortungssysteme an, um diesen sicherheitsrelevanten Bereich mit einer technischen Lösung zu versorgen. Die Bandbreite reicht dabei vom handelsüblichen Smartphone mit Ortungs-App bis hin zu weitaus aufwändigeren und vor allem größeren Devices für das Handgelenk, den Gurt oder den Schuh (Megges et al., 2014 (a)). Die technischen Angebote geben an, sich bewusst an den speziellen Bedürfnissen des Menschen mit Demenz und dessen Angehörigen zu orientieren. Unsere Nutzerstudien identifizierten jedoch zusammengefasst drei wesentliche Herausforderungen (Megges et al. 2013 (a) u. 2014 (b)):

- zu hoher Energieverbrauch der Geräte,

- mangelhafte Benutzerfreundlichkeit (Usability) der Hardware sowie

- Ungenauigkeit bzw. Fehleranfälligkeit der Ortung.

Insofern besteht eine enorme Diskrepanz zwischen dem auf der einen Seite bestehenden, hohen Bedarf und der auf der anderen Seite ungenügenden Produktpalette.

\subsection{Fragestellung}

Abgeleitet aus den bisherigen Ergebnissen und Studien ergibt sich für das vorliegende Forschungsprojekt in einem ersten Schritt die Frage, anhand welcher Kriterien Menschen mit Demenz und deren Angehörige im häuslichen Bereich identifiziert werden können, die von einem bestimmten Ortungssystem profitieren? Für die praktische Anwendung soll eine Checkliste entwickelt werden, die anhand gezielter Fragen in der Praxis Tätigen mit der Zielgruppe hilft, konkrete Empfehlungen auszusprechen. Das Ziel ist es potenziellen Nutzern das Produkt mit dem antizipierten größten individuellen Nutzen vorzuschlagen.

\section{Methodik}

Das Vorhaben zeichnet sich durch einen multimethodalen (mixed methods) Ansatz aus. Da die Anwender von Ortungssystemen bei Demenz eine stark eingrenzbare Grundgesamtheit darstellen, lassen sich die spezifischen Fragen am besten in einer Kombination von qualitativen und quantitativen Methoden beantworten. Diese Vorgehensweise gilt in diesem Bereich als Best Practice Methode. Um die Checkliste zu erstellen, müssen verschiedene Schritte erfolgen. Zunächst wird eine standardisierte Produktrecherche im Internet durchgeführt. Die Produktrecherche fand systematisiert und mithilfe vordefinierter Kriterien statt. Um praxisnahe Ergebnisse $\mathrm{zu}$ erhalten, erfolgte die Suche nach personenbezogenen Ortungssystemen im Internet, mithilfe der Suchmaschine Google. Initial wurde für den deutschsprachigen Raum nach den Begriffen „Ortung“ und „Demenz“ gesucht. Von den 
rund 15.400 Suchergebnissen wurden die als relevant eingestuften Ergebnisse ${ }^{1}$ gesichtet und die Produkte in einer Übersicht aufgenommen. Auch Werbeanzeigen wurden dabei berücksichtigt und angezeigte Foren auf Produktnennungen hin überprüft. Anschließend wurden die o.g. Suchbegriffe mit weiteren Begriffen wie „GPS“ - Global Positioning System oder „Alzheimer“ kombiniert. Darüber hinaus erfolgte eine englischsprachige Suche, unter anderem nach den Begriffen „,dementia“ und ,tracking“. Die recherchierten Produkte wurden dabei auf bestimmte Kriterien hin untersucht, und die Ergebnisse anschließend in einem Kriterienkatalog zusammengetragen und von Experten bewertet. Die am höchsten gerankten und bereits in wissenschaftlichen Arbeiten evaluierten Produkte für die Zielgruppe wurden ausgewählt und für umfangreiche Praxistests angeschafft (Einrichtung der Ortungssysteme, Usability, etc.). Hier fanden sowohl Experten- als auch Nutzerbefragungen statt. Methodisch wurden Interviewleitfäden entwickelt sowie standardisierte Fragebögen (fünf-stufige Likertskalen) zur Produktbewertung verwendet. Außerdem werden zur besseren Beschreibung der Zielgruppe wissenschaftliche Erkenntnisse herangezogen, die auf verschiedene Krankheitsstadien mit unterschiedlichen Symptomen eingehen sowie vor allem auf die Einschränkungen der Menschen mit dem Demenz in Bezug auf Mobilität und alltagspraktische Fähigkeiten (Lawton \& Brody, 1969, Bucks et al., 1996).

\section{Ergebnisse}

Durch die Produktrecherche wurden sieben Bereiche identifiziert, welche die wesentlichen Merkmale der am Markt befindlichen Ortungssysteme beschreiben. Die Bereiche sind: Produktmerkmale, Technik, Funktion, Regularien, Service und Informationen, Kosten und Praxistest. Darüber hinaus wurde eine abschließende Zusammenfassung verfasst. Die folgende Tabelle 1 stellt einen Auszug des Kriterienkataloges dar.

\begin{tabular}{|l|l|l|l|}
\hline Produkt & Produktmerkmale & Technik & Funktion \\
\hline \multirow{3}{*}{ Produkt 1} & Anbringung am Körper & Touchscreen vorhanden & $\begin{array}{l}\text { Darstellungsform der } \\
\text { Ortung }\end{array}$ \\
\cline { 2 - 4 } & Gewicht & $\begin{array}{l}\text { Signalgeber (LEDs) } \\
\text { vorhanden }\end{array}$ & $\begin{array}{l}\text { Notruffunktion für } \\
\text { Menschen mit Demenz }\end{array}$ \\
\cline { 2 - 4 } & $\begin{array}{l}\text { Sicherheitsverschluss } \\
\text { vorhanden }\end{array}$ & $\begin{array}{l}\text { Akkutechnik und } \\
\text { Akkulaufzeit }\end{array}$ & $\begin{array}{l}\text { Notrufunabhängige } \\
\text { Ortung für Angehörige }\end{array}$ \\
\hline
\end{tabular}
1 Als relevant galten diejenigen Suchergebnisse, die in der Google-Vorschau einen Bezug zum Untersuchungs-
gegenstand aufwiesen. 


\begin{tabular}{|l|l|l|l|}
\hline \multirow{2}{*}{} & Tragekomfort & $\begin{array}{l}\text { Ortungstechnik- und - } \\
\text { intervall }\end{array}$ & $\begin{array}{l}\text { Geofencing- } \\
\text { Konfiguration }\end{array}$ \\
\cline { 2 - 4 } & Verwendete Materialien & Anschlüsse & $\begin{array}{l}\text { Erfahrungen bei der } \\
\text { Nutzung }\end{array}$ \\
\hline
\end{tabular}

Tabelle 1: Auszug aus Kriterienkatalog

Die angebotenen Produkte unterscheiden sich in Kriterien wie der Ortungsmethode, der Interaktionsmöglichkeit zwischen Träger und Angehörigem, sowie der Anbringungsart am Körper. Aber auch bei den einmaligen und laufenden Kosten gibt es teils erhebliche Unterschiede. Im Weiteren zeigt sich, dass die Anbieter respektive Hersteller nicht immer ausschließlich den Menschen mit Demenz und dessen Angehörige als Zielgruppe im Fokus haben. Oftmals sprechen die Produkte vulnerable Zielgruppen an (Menschen mit Autismus, mit körperlichen und/oder geistigen Einschränkungen sowie Kinder) aber auch und vor allem Gegenstände (PKWs, Wertgegenstände, etc.).

\section{Diskussion und Ausblick}

Die Untersuchung der Produkte lässt erkennen, inwieweit die Systeme sich unterscheiden und welchen Einfluss die Gestaltung spezifischer funktionaler wie nicht funktionaler Merkmale auf die Produkteigenschaften hat. Dies übt letztlich einen großen Einfluss auf die Nutzerfreundlichkeit und somit auf das Nutzererlebnis mit dem jeweiligen Produkt aus. Im Sinne des Nutzererlebnisses wird deutlich wie wichtig es ist, beide Teile der Dyade, also sowohl den Menschen mit Demenz als auch dessen Angehörigen in alle Überlegungen hinsichtlich der Nutzung und Optimierung miteinzubeziehen. Dies bedeutet, den Erkrankten mit seinen spezifischen Bedürfnissen, seinen teils sehr unterschiedlich ausgeprägten Krankheitssymptomen, seinen individuellen Verhaltensweisen und seinen Gewohnheiten zu berücksichtigen und gar in den Vordergrund zu stellen.

In mehreren Entwicklungs- und Umsetzungsschleifen greift das Forschungsprojekt das Thema des Nutzererlebnisses insbesondere dadurch auf, dass es durch die Nähe zum Anwender von ihm lernt. Um die tatsächliche Nutzung in der Praxis zu gewährleisten, bedarf es einer angepassten Heranführungsweise. Gemeint sind damit spezielle Schulungskonzepte sowie optimierte Bedienungsanleitungen, die das Nutzererlebnis vor und während der Anwendung maßgeblich beeinflussen. In einem nächsten Schritt werden daher Schulungskonzepte entwickelt sowie Nutzertests im Alltag der Zielgruppe durchgeführt. Dafür werden verschiedene Module erstellt, die insbesondere in der Anfangsphase der

2 Geofencing beschreibt die Funktion, einen Bereich zu markieren. Beim Verlassen oder Betreten dieses Bereiches wird ein automatisierter Alarm ausgelöst. Somit können Angehörige beispielsweise alarmiert werden, wenn der Mensch mit Demenz sich einer Gefahrenzone nähert. 
Nutzung der Hilfen eine engmaschige Begleitung der Anwender gewährleisten sollen. Auch an dieser Stelle steht die Einbindung beider Akteure der Dyade im Vordergrund. Die Einbindung des Menschen mit Demenz hat trotz oder gerade wegen seiner Erkrankung einen besonderen Stellenwert für das weitere Projekt.

Im Ergebnis möchte unsere Forschungsgruppe die beschriebenen Hürden überwunden haben und das Nutzererlebnis der Menschen mit Demenz und deren Angehörigen mit technischen Hilfen nachhaltig positiv beeinflussen. Sicherheit, Unabhängigkeit und die Stärkung der Individualität der Menschen sind ein hohes Gut, welches es zu bewahren gilt.

\section{Danksagung}

Ein besonderer Dank gilt der Deutschen Alzheimergesellschaft, welche dieses Forschungsprojekt ermöglicht.

\section{Literaturverzeichnis}

Berlin-Institut für Bevölkerung und Entwicklung (Hg.) (2011). Demenz-Report. Wie sich die Regionen in Deutschland, Österreich und der Schweiz auf die Alterung der Gesellschaft vorbereiten können. Gebrüder-Kopp: Köln.

Braun, M., \& Scholz, U. H. R. \&. M. M. (2010). Die subjektive Belastung pflegender Ehepartner von Demenzerkrankten: Hinweise zur Validität des deutschen Zarit Burden Interviews. Zeitschrift für Gerontologie und Geriatrie, 43, 111-119.

Bucks, R. S., Ashworth, D. L., Wilcock, G. K., \& Siegfried, K. (1996). Assessment of Activities of Daily Living in Dementia: Development of the Bristol Activities of Daily Living Scale. Age and Ageing, 25(2), 113-120.

Kinney, J. M., Kart, C. S., Murdoch, L. D., \& Ziemba, T. F. (2003). Challenges in caregiving and creative solutions: Using technology to facilitate caring for a relative with dementia. Ageing International, 28(3), 295-313.

Lawton, M. P., Brody, E. M. (1969). Assessment of older people: Self-maintaining and instrumental activities of daily living. The Gerontologist, 9(3), 179-186.

Megges, H., Peters, O. (2014). Wo bist du? Nutzereinbindung in die Entwicklung technischer Assistenzen bei Demenz 10. World Usability Day, Berlin, November, 2014 (a).

Megges, H., Jankowski, N., Geissler, L., Fasold, U.-G., Peters, O. (2014). Ein technisches Assistenzsystem auf dem Prüfstand - Ergebnisse einer Nutzerstudie mit pflegenden Angehörigen. $B M B F, V D E$ e.V., AAL Association, \& VDE/VDE/IT (Eds.), Wohnen - Pflege - Teilhabe - Besser leben durch Technik, AAL 2014 (b).

Megges, H., Jankowski, N., Peters, O. (2013). Caregiver needs analysis for product development of an assistive technology system in dementia care. 23th Alzheimer Europe Conference, St. Julian's, Malta, October, 2013 (a).

Megges, H., Geissler, L., Fasold, U.-G., Peters, O. (2013). Nutzerpartizipation in der Produktentwicklung eines technischen Assistenzsystems für Pflegende von Demenzkranken. $B M B F, V D E$ e.V., AAL Association, \& VDE/VDE/IT (Eds.), Lebensqualität im Wandel von Demografie und Technik, AAL 2013 (b). 
Vuong, N.K., Chan, S., Lau, C.T. (2015). mHealth Sensors, Techniques, and Applications for Managing Wandering Behavior of People with Dementia: A Review. Mobile Health Springer Series in Bio-/Neuroinformatics Volume 5, 2015, pp 11-42.

Wan, L.; Müller, C.; Wulf, V.; Randall, D. (2014). Addressing the Subtleties in Dementia Care: Prestudy \& Evaluation of a GPS Monitoring System. Session: Network of Care CHI 2014, One of a CHInd, Toronto, ON, Canada.

Weinberger, N., Decker, M., Krings, B. (2013). Pflege von Menschen mit Demenz - Bedarfsorientierte Technikgestaltung, In: Schultz, T., Putze, F., Kruse, A. (Hrsg.) Technische Unterstützung für Menschen mit Demenz KIT Scientific Publishing: pp.61-74.

Sapmaz, E.; Reichwaldt, N.; Haux, R. (2012). Einsatz mobiler Endgeräte im Bereich Personenortung bei dementiell erkrankten Menschen - Durchführung einer Studie zur Evaluation geeigneter mobiler Endgeräte. Poster des Peter L. Reichertz Institut für Medizinische Informatik der Technischen Universität Braunschweig und der Medizinischen Hochschule Hannover.

Sapmaz, E. (2012). Einsatz mobiler Endgeräte im Bereich Personenortung bei dementiell erkrankten Menschen. Nicht veröffentlichte Diplomarbeit vorgelegt am Peter L. Reichertz Institut für Medizinische Informatik der Technischen Universität Braunschweig und der Medizinischen Hochschule Hannover.

Schneekloth, U., \& Wahl, H.-W. (Eds.) (2005). Möglichkeiten und Grenzen selbständiger Lebensführung in privaten Haushalten (MuG III): Repräsentativbefunde und Vertiefungsstudien zu häuslichen Pflegearrangements, Demenz und professionellen Versorgungsangeboten. München.

\section{Kontaktinformationen}

Herlind Megges, Torsten Grewe, Oliver Peters

Charité Universitätsmedizin Berlin, Klinik für Psychiatrie, Modul Altersmedizin

Lindenberger Weg 80, 13125 Berlin, Deutschland

\{herlind.megges, torsten.grewe, oliver.peters\}@ charite.de

http://www.psychiatrie.charite.de/klinik/module/altersmedizin/forschung/ 
